\title{
Online Appendix: Does school desegregation promote diverse interactions? An equilibrium model of segregation within schools
}

\author{
By Angelo Mele
}

\section{Computational Details}

The linear specification allows for utility functions involving network-level controls, when estimation is performed using multiple networks. This can be achieved by a specification of the parameters such as

$$
\theta_{p}=\theta_{p 0}+\sum_{c=1}^{C} \theta_{p c} Z_{c}
$$

where $Z_{c}$ is a network-level variable. The estimation methodology presented above can be applied to this specification without any change. However, estimation of a model with unobserved heterogeneity would require significant additional computational effort (see Appendix C in Mele (2017)).

I choose somewhat vague priors for the parameters to extract most of the information from the data. I assume independent normal priors

$$
p(\theta)=\mathcal{N}\left(\mathbf{0}, 3 \mathbf{I}_{P}\right),
$$

where $P$ is the number of parameters.

The proposal distribution for the posterior simulation is

$$
q_{\theta}(\cdot \mid \theta)=\mathcal{N}(\mathbf{0}, \delta \boldsymbol{\Sigma})
$$

where $\delta$ is a scaling factor and $\boldsymbol{\Sigma}$ is a covariance matrix.

I use an adaptive procedure to determine a suitable $\boldsymbol{\Sigma}$. I start the iterations with $\boldsymbol{\Sigma}=\lambda \mathbf{I}_{P}$, where $\lambda$ is a vector of standard deviations. I choose $\lambda$ so that the sampler accepts at least 20\%-25\% of the proposed parameters, as is standard in the literature (Gelman et al., 2003; Robert and Casella, 2005). I run the chain and monitor convergence using standard methods. Once the chains have reached approximate convergence, I estimate the covariance matrix of the chains and use it as an approximate $\boldsymbol{\Sigma}$ for the next set of simulations. The scaling factor is $\delta=2.38^{2} / P$ as suggested in Gelman, Roberts and Gilks (1996).

The network sampler uses a proposal $q_{g}\left(g \mid g^{\prime}\right)$, that selects a link to be updated

$$
1
$$


at each period according to a discrete uniform distribution. The probability of network inversion is $p_{i n v}=0.01$.

All the posterior distributions shown in the following graphs are obtained with a simulation of 100000 Metropolis-Hastings updates of the parameters. These simulations start from values found after extensive experimentation with different starting values and burn-in periods, monitoring convergence using standard methods. For each parameter update, I simulate the network for 5000 iterations to collect a sample from the stationary distribution. For the estimation with 16 schools I ran 20000 parameter simulations and 10000 network simulations for each parameter.

\section{A1. Parallel estimation with multiple networks}

When data from multiple independent networks are available the estimation routines are easily adapted. Assume the researcher has data from $C$ networks: let $g_{c}$ and $X_{c}$ denote the network matrix and the individual controls for network $c, c=1, \ldots, C$. The aggregate data are denoted as $g=\left\{g_{1}, \ldots, g_{c}\right\}$ and $X=$ $\left\{X_{1}, \ldots, X_{c}\right\}$.

Assuming each network is drawn from the stationary equilibrium of the model, each network has distribution

$$
\pi\left(g_{c}, X_{c}, \theta\right)=\frac{\exp \left[Q\left(g_{c}, X_{c}, \theta\right)\right]}{\sum_{\omega \in \mathcal{G}_{c}} \exp \left[Q\left(\omega_{c}, X_{c}, \theta\right)\right]}
$$

Since each network is independent, the likelihood of the data $(g, X)$ can be written as

$$
\begin{aligned}
\pi(g, X, \theta) & =\prod_{c=1}^{C} \pi\left(g_{c}, X_{c}, \theta\right)=\prod_{c=1}^{C}\left\{\frac{\exp \left[Q\left(g_{c}, X_{c}, \theta\right)\right]}{c\left(\mathcal{G}_{c}, X_{c}, \theta\right)}\right\} \\
& =\frac{\exp \left[\sum_{c=1}^{C} Q\left(g_{c}, X_{c}, \theta\right)\right]}{\prod_{c=1}^{C} c\left(\mathcal{G}_{c}, X_{c}, \theta\right)}=\frac{\exp \left[\sum_{c=1}^{C} Q\left(g_{c}, X_{c}, \theta\right)\right]}{\mathcal{C}(\mathcal{G}, X, \theta)}
\end{aligned}
$$

where $\mathcal{G}=\bigcup_{c=1}^{C} \mathcal{G}_{c}$ and $X=\left\{X_{1}, \ldots, X_{C}\right\}$. The likelihood for multiple independent networks is of the same form as the likelihood for one network observation. The structure of this likelihood makes parallelization extremely easy: each network can be simulated independently using the network simulation algorithm; at the end of the simulation we collect the last network and compute the potential; then we compute the sum of potentials and use it to compute the probability of update.

Therefore, the algorithm is modified as follows

\section{ALGORITHM 1: (Parallel approximate exchange algorithm}


Fix the number of simulations $R$. Store each network data $\left(g_{c}, X_{c}\right)$ in a different processor/core. At each iteration $t$, with current parameter $\theta_{t}=\theta$ and network data $g$

1) Propose a new parameter $\theta^{\prime}$ from a distribution $q_{\theta}(\cdot \mid \theta)$

$$
\theta^{\prime} \sim q_{\theta}(\cdot \mid \theta)
$$

2) For each processor $c$, start the network sampler at the observed network $g_{c}$, iterating for $R$ steps using parameter $\theta^{\prime}$ and collect the last simulated network $g_{c}^{\prime}$

$$
g_{c}^{\prime} \sim \mathcal{P}_{\theta^{\prime}}^{(R)}\left(g_{c}^{\prime} \mid g_{c}\right)
$$

3) Update the parameter according to

$$
\theta_{t+1}= \begin{cases}\theta^{\prime} & \text { with prob. } \alpha_{\text {pex }}\left(\theta, \theta^{\prime}\right) \\ \theta & \text { with prob. } 1-\alpha_{\text {pex }}\left(\theta, \theta^{\prime}\right)\end{cases}
$$

where

$$
\left.\alpha_{p e x}\left(\theta, \theta^{\prime}\right)=\operatorname{mi(nA}\{) 1, \frac{\exp \left[\sum_{c=1}^{C} Q\left(g_{c}^{\prime}, X_{c}, \theta\right)\right]}{\exp \left[\sum_{c=1}^{C} Q\left(g_{c}, X_{c}, \theta\right)\right]} \frac{p\left(\theta^{\prime}\right)}{p(\theta)} \frac{q_{\theta}\left(\theta \mid \theta^{\prime}\right)}{q_{\theta}\left(\theta^{\prime} \mid \theta\right)} \frac{\exp \left[\sum_{c=1}^{C} Q\left(g_{c}, X_{c}, \theta^{\prime}\right)\right]}{\exp \left[\sum_{c=1}^{C} Q\left(g_{c}^{\prime}, X_{c}, \theta^{\prime}\right)\right]}\right\}
$$

The speed of the algorithm depends on the largest network in the data. Since each parameter update requires the result of each processor simulation there is some idle time, since small networks are simulated much faster. However, one could easily modify the algorithm to have different number of network simulations for networks of different sizes, so for each $c$ we would have a different $R_{c}$

\section{Freeman Segregation Index}

The Freeman segregation index measures the degree of segregation in a population with two groups (Freeman, 1972). Assume there are two groups, A and B. Let $n_{A B}$ be the total number of links that individuals of group $\mathrm{A}$ form to individuals of group B. Let $n_{B A}, n_{B B}$ and $n_{A A}$ be analogously defined. The original index developed by Freeman (1972) is defined as

$$
F S I=\frac{\mathbb{E}\left[n_{A B}\right]+\mathbb{E}\left[n_{B A}\right]-\left(n_{A B}+n_{B A}\right)}{\mathbb{E}\left[n_{A B}\right]+\mathbb{E}\left[n_{B A}\right]}
$$

When the link formation does not depend on the identity of individuals, then the links should be randomly distributed with respect to identity. Therefore, the 
index measures the difference between the expected and actual number of links among individuals of different groups, as a fraction of the expected links. An index of 0 means that the actual network closely resembles one in which links are formed at random. Higher values indicate more segregation. In this paper segregation is measured using the index ${ }^{1}$

$$
S E G=\max \{0, F S I\}
$$

The index varies between 0 and 1, where the maximum corresponds to a network in which there are no cross-group links.

To complete the derivation of the index, the expected number of cross-group links is computed as

$$
\begin{aligned}
& \mathbb{E}\left[n_{A B}\right]=\frac{\left(n_{A A}+n_{A B}\right)\left(n_{A B}+n_{B B}\right)}{n_{A A}+n_{A B}+n_{B A}+n_{B B}} \\
& \mathbb{E}\left[n_{B A}\right]=\frac{\left(n_{B A}+n_{B B}\right)\left(n_{A A}+n_{B A}\right)}{n_{A A}+n_{A B}+n_{B A}+n_{B B}}
\end{aligned}
$$

\section{REFERENCES}

Freeman, L. 1972. "Segregation in social networks." Sociological Methods and Research, 6: 411-427.

Gelman, A., G. O. Roberts, and W. R. Gilks. 1996. "Efficient Metropolis jumping rules." Bayesian Statistics, 5: 599-608.

Gelman, A., J. Carlin, H. Stern, and D. Rubin. 2003. Bayesian Data Analysis, Second Edition. Chapman \& Hall/CRC.

Mele, Angelo. 2017. "A Structural Model of Dense Network Formation." Econometrica, 85: 825-850.

Robert, Christian P., and George Casella. 2005. Monte Carlo Statistical Methods (Springer Texts in Statistics). Secaucus, NJ, USA:Springer-Verlag New York, Inc.

\section{Posterior estimates (COMPlete tables)}

\footnotetext{
${ }^{1}$ The index (B1) varies between -1 and 1 . However, the interpretation of the index when it assumes negative values is not clear. Therefore Freeman (1972) suggests to use only when it is nonnegative, to measure the presence of segregation
} 
Table D1-Model (2) in Table 3

\begin{tabular}{|c|c|c|c|c|c|}
\hline & mean & median & std. dev. & 5 pctile & 95 pctile \\
\hline \multicolumn{6}{|c|}{ A. DiRECT UTILITY $\left(u_{i j}\right)$} \\
\hline CONSTANT & -5.5381 & -5.5384 & 0.0261 & -5.5805 & -5.4942 \\
\hline SAME GENDER & 0.1850 & 0.1850 & 0.0133 & 0.1631 & 0.2069 \\
\hline SAME GRADE & 2.2384 & 2.2384 & 0.0030 & 2.2334 & 2.2434 \\
\hline WHITE-WHITE & 0.5414 & 0.5413 & 0.0048 & 0.5335 & 0.5494 \\
\hline BLACK-BLACK & 0.3660 & 0.3661 & 0.0173 & 0.3375 & 0.3943 \\
\hline HISP-HISP & 1.6794 & 1.6794 & 0.0322 & 1.6267 & 1.7324 \\
\hline ATTRACTIVE i (Physical) & 0.3068 & 0.3070 & 0.0270 & 0.2623 & 0.3509 \\
\hline ATTRACTIVE j (Physical) & 0.2322 & 0.2322 & 0.0076 & 0.2198 & 0.2448 \\
\hline ATTRACTIVE i (Personality) & 0.0063 & 0.0061 & 0.0128 & -0.0145 & 0.0275 \\
\hline ATTRACTIVE j (Personality) & 0.8678 & 0.8679 & 0.0173 & 0.8391 & 0.8959 \\
\hline Income i - Income j (logs) & 0.1462 & 0.1461 & 0.0068 & 0.1351 & 0.1574 \\
\hline Income $\mathrm{i}+$ Income $\mathrm{j}(\operatorname{logs})$ & -0.0806 & -0.0806 & 0.0049 & -0.0885 & -0.0725 \\
\hline FRACTION WHITES & -0.4814 & -0.4814 & 0.0320 & -0.5338 & -0.4284 \\
\hline FRACTION BLACKS & 3.0985 & 3.0984 & 0.0156 & 3.0730 & 3.1242 \\
\hline FRACTION HISP & 2.4440 & 2.4439 & 0.0207 & 2.4100 & 2.4781 \\
\hline WHITE-WHITE * FRACTION WHITES & 1.0094 & 1.0095 & 0.0267 & 0.9659 & 1.0532 \\
\hline BLACK-BLACK $*$ FRACTION BLACKS & 0.1478 & 0.1478 & 0.0095 & 0.1322 & 0.1633 \\
\hline HISP-HISP $*$ FRACTION HISP & -1.4255 & -1.4258 & 0.0309 & -1.4758 & -1.3744 \\
\hline SCHOOL 1 & -2.1181 & -2.1186 & 0.0616 & -2.2192 & -2.0164 \\
\hline SCHOOL 2 & 1.5065 & 1.5066 & 0.0105 & 1.4891 & 1.5236 \\
\hline SCHOOL 3 & -0.0532 & -0.0531 & 0.0299 & -0.1022 & -0.0040 \\
\hline SCHOOL 4 & 1.1044 & 1.1043 & 0.0154 & 1.0793 & 1.1297 \\
\hline SCHOOL 5 & 1.3247 & 1.3246 & 0.0091 & 1.3098 & 1.3397 \\
\hline SCHOOL 6 & 0.4666 & 0.4667 & 0.0224 & 0.4300 & 0.5037 \\
\hline SCHOOL 7 & 2.3742 & 2.3741 & 0.0261 & 2.3313 & 2.4171 \\
\hline SCHOOL 8 & 0.2750 & 0.2750 & 0.0382 & 0.2125 & 0.3374 \\
\hline SCHOOL 9 & -1.3631 & -1.3631 & 0.0281 & -1.4090 & -1.3171 \\
\hline SCHOOL 10 & 1.7814 & 1.7817 & 0.0339 & 1.7255 & 1.8369 \\
\hline SCHOOL 11 & -1.4060 & -1.4059 & 0.0094 & -1.4216 & -1.3905 \\
\hline SCHOOL 12 & 2.9026 & 2.9027 & 0.0241 & 2.8627 & 2.9422 \\
\hline SCHOOL 13 & 0.3076 & 0.3076 & 0.0420 & 0.2388 & 0.3770 \\
\hline \multicolumn{6}{|c|}{$\underline{\text { B. Mutual utility }}\left(m_{i j}\right)$} \\
\hline CONSTANT & 1.1853 & 1.1852 & 0.0388 & 1.1218 & 1.2484 \\
\hline SAME GENDER & 1.1652 & 1.1653 & 0.0121 & 1.1452 & 1.1849 \\
\hline SAME GRADE & -1.6882 & -1.6883 & 0.0210 & -1.7228 & -1.6537 \\
\hline WHITE-WHITE & 0.0073 & 0.0074 & 0.0132 & -0.0147 & 0.0289 \\
\hline BLACK-BLACK & 0.7468 & 0.7468 & 0.0318 & 0.6943 & 0.7992 \\
\hline HISP-HISP & 0.7779 & 0.7778 & 0.0089 & 0.7635 & 0.7925 \\
\hline \multicolumn{6}{|c|}{ C. INDIRECT UTILITY AND POPULARITY $\left(v_{i j}\right)$} \\
\hline CONSTANT & -0.2891 & -0.2890 & 0.0129 & -0.3105 & -0.2680 \\
\hline SAME GENDER & 0.1721 & 0.1721 & 0.0053 & 0.1635 & 0.1808 \\
\hline SAME GRADE & -0.3145 & -0.3145 & 0.0066 & -0.3254 & -0.3038 \\
\hline WHITE-WHITE & 0.2239 & 0.2238 & 0.0085 & 0.2099 & 0.2379 \\
\hline BLACK-BLACK & -0.1364 & -0.1364 & 0.0146 & -0.1605 & -0.1124 \\
\hline HISP-HISP & 0.4328 & 0.4327 & 0.0105 & 0.4157 & 0.4501 \\
\hline
\end{tabular}

Estimated posterior distribution for the full structural model. The estimates are obtained with a sample of 100000 parameter simulations, and 5000 network simulations for each parameter proposal. 
Table D2-Model (1) in Table 3

\begin{tabular}{|c|c|c|c|c|c|}
\hline & mean & median & std. dev. & 5 pctile & 95 pctile \\
\hline CONSTANT & -6.9201 & -6.9196 & 0.0393 & -6.9854 & -6.8562 \\
\hline SAME GENDER & -0.4545 & -0.4546 & 0.0204 & -0.4884 & -0.4213 \\
\hline SAME GRADE & 2.3124 & 2.3124 & 0.0030 & 2.3075 & 2.3174 \\
\hline WHITE-WHITE & 0.3504 & 0.3505 & 0.0061 & 0.3402 & 0.3603 \\
\hline BLACK-BLACK & 0.1443 & 0.1444 & 0.0183 & 0.1135 & 0.1742 \\
\hline HISP-HISP & 1.8597 & 1.8598 & 0.0297 & 1.8109 & 1.9085 \\
\hline ATTRACTIVE i (Physical) & 0.2757 & 0.2760 & 0.0267 & 0.2314 & 0.3191 \\
\hline ATTRACTIVE j (Physical) & -0.0410 & -0.0411 & 0.0104 & -0.0583 & -0.0241 \\
\hline ATTRACTIVE i (Personality) & -0.4402 & -0.4399 & 0.0152 & -0.4657 & -0.4158 \\
\hline ATTRACTIVE j (Personality) & 1.0672 & 1.0672 & 0.0179 & 1.0378 & 1.0966 \\
\hline Income i - Income j (logs) & 0.1793 & 0.1792 & 0.0071 & 0.1676 & 0.1911 \\
\hline Income $\mathrm{i}+$ Income $\mathrm{j}(\operatorname{logs})$ & -0.0882 & -0.0882 & 0.0050 & -0.0963 & -0.0800 \\
\hline FRACTION WHITES & 0.9070 & 0.9067 & 0.0465 & 0.8312 & 0.9843 \\
\hline FRACTION BLACKS & 3.2238 & 3.2237 & 0.0153 & 3.1989 & 3.2491 \\
\hline FRACTION HISP & 2.5240 & 2.5237 & 0.0211 & 2.4900 & 2.5593 \\
\hline WHITE-WHITE * FRACTION WHITES & 1.3962 & 1.3959 & 0.0271 & 1.3526 & 1.4409 \\
\hline BLACK-BLACK * FRACTION BLACKS & 0.4664 & 0.4665 & 0.0127 & 0.4457 & 0.4875 \\
\hline HISP-HISP $*$ FRACTION HISP & -1.5643 & -1.5643 & 0.0305 & -1.6135 & -1.5135 \\
\hline SCHOOL 1 & -3.4873 & -3.4871 & 0.0739 & -3.6103 & -3.3653 \\
\hline SCHOOL 2 & 1.8278 & 1.8278 & 0.0115 & 1.8089 & 1.8469 \\
\hline SCHOOL 3 & -0.5626 & -0.5623 & 0.0317 & -0.6159 & -0.5110 \\
\hline SCHOOL 4 & 0.4159 & 0.4159 & 0.0219 & 0.3796 & 0.4516 \\
\hline SCHOOL 5 & 1.4366 & 1.4365 & 0.0082 & 1.4232 & 1.4503 \\
\hline SCHOOL 6 & 1.3884 & 1.3882 & 0.0311 & 1.3376 & 1.4399 \\
\hline SCHOOL 7 & 2.8597 & 2.8592 & 0.0283 & 2.8139 & 2.9071 \\
\hline SCHOOL 8 & 1.2675 & 1.2672 & 0.0446 & 1.1948 & 1.3421 \\
\hline SCHOOL 9 & -1.9436 & -1.9431 & 0.0306 & -1.9940 & -1.8944 \\
\hline SCHOOL 10 & 1.7678 & 1.7679 & 0.0352 & 1.7091 & 1.8254 \\
\hline SCHOOL 11 & -0.9222 & -0.9224 & 0.0144 & -0.9455 & -0.8981 \\
\hline SCHOOL 12 & 3.5492 & 3.5491 & 0.0297 & 3.5004 & 3.5986 \\
\hline SCHOOL 13 & -0.3995 & -0.3996 & 0.0445 & -0.4720 & -0.3257 \\
\hline
\end{tabular}


Table D3-Model (4) in TABle 3

\begin{tabular}{|c|c|c|c|c|c|}
\hline & mean & median & std. dev. & 5 pctile & 95 pctile \\
\hline \multicolumn{6}{|c|}{ A. DiRECT UTILITY $\left(u_{i j}\right)$} \\
\hline CONSTANT & -5.9132 & -5.9131 & 0.0146 & -5.9373 & -5.8894 \\
\hline MALE & 0.0463 & 0.0464 & 0.0119 & 0.0264 & 0.0657 \\
\hline WHITE & 0.0044 & 0.0045 & 0.0163 & -0.0224 & 0.0310 \\
\hline BLACK & 1.1310 & 1.1311 & 0.0063 & 1.1208 & 1.1414 \\
\hline HISP & 2.2806 & 2.2804 & 0.0303 & 2.2308 & 2.3306 \\
\hline INCOME & -1.6492 & -1.6490 & 0.0381 & -1.7122 & -1.5869 \\
\hline SAME GENDER & 0.4851 & 0.4851 & 0.0155 & 0.4597 & 0.5107 \\
\hline SAME GRADE & 2.0113 & 2.0113 & 0.0264 & 1.9674 & 2.0545 \\
\hline WHITE-WHITE & 0.5720 & 0.5719 & 0.0129 & 0.5510 & 0.5933 \\
\hline BLACK-BLACK & 1.1445 & 1.1442 & 0.0147 & 1.1208 & 1.1691 \\
\hline HISP-HISP & -0.2269 & -0.2270 & 0.0172 & -0.2553 & -0.1986 \\
\hline BEAUTY i & -2.2413 & -2.2411 & 0.0382 & -2.3044 & -2.1790 \\
\hline BEAUTY j & 1.5861 & 1.5857 & 0.0207 & 1.5525 & 1.6204 \\
\hline PERSONALITY i & -0.1570 & -0.1570 & 0.0100 & -0.1736 & -0.1404 \\
\hline PERSONALITY j & -0.7390 & -0.7388 & 0.0185 & -0.7698 & -0.7088 \\
\hline Income $\mathrm{i}$ - Income $\mathrm{j}$ & 0.9012 & 0.9010 & 0.0208 & 0.8672 & 0.9356 \\
\hline Income i + Income j & 0.9244 & 0.9242 & 0.0220 & 0.8885 & 0.9607 \\
\hline FRACTION WHITES & -1.4420 & -1.4420 & 0.0091 & -1.4569 & -1.4269 \\
\hline FRACTION BLACKS & 1.8309 & 1.8309 & 0.0119 & 1.8114 & 1.8504 \\
\hline FRACTION HISP & 0.7798 & 0.7798 & 0.0106 & 0.7624 & 0.7970 \\
\hline WHITE-WHITE * FRACTION WHITE & 2.7840 & 2.7831 & 0.0504 & 2.7028 & 2.8685 \\
\hline BLACK-BLACK $*$ FRACTION BLACKS & 0.4028 & 0.4028 & 0.0063 & 0.3923 & 0.4130 \\
\hline HISP-HISP $*$ FRACTION HISP & -1.3630 & -1.3629 & 0.0075 & -1.3754 & -1.3508 \\
\hline SCHOOL 1 & -0.0766 & -0.0766 & 0.0279 & -0.1220 & -0.0307 \\
\hline SCHOOL 2 & 1.3889 & 1.3890 & 0.0273 & 1.3436 & 1.4337 \\
\hline SCHOOL 3 & 1.8308 & 1.8306 & 0.0185 & 1.8005 & 1.8610 \\
\hline SCHOOL 4 & 1.4277 & 1.4276 & 0.0173 & 1.3996 & 1.4565 \\
\hline SCHOOL 5 & 1.9201 & 1.9201 & 0.0145 & 1.8961 & 1.9440 \\
\hline SCHOOL 6 & -0.7518 & -0.7519 & 0.0197 & -0.7841 & -0.7191 \\
\hline SCHOOL 7 & 0.0355 & 0.0355 & 0.0135 & 0.0129 & 0.0576 \\
\hline SCHOOL 8 & -0.5121 & -0.5122 & 0.0228 & -0.5494 & -0.4746 \\
\hline SCHOOL 9 & -2.6615 & -2.6613 & 0.0559 & -2.7538 & -2.5701 \\
\hline SCHOOL 10 & 1.1371 & 1.1374 & 0.0345 & 1.0796 & 1.1937 \\
\hline SCHOOL 11 & -0.8724 & -0.8725 & 0.0274 & -0.9173 & -0.8271 \\
\hline SCHOOL 12 & 1.6418 & 1.6419 & 0.0207 & 1.6078 & 1.6758 \\
\hline SCHOOL 13 & 1.3257 & 1.3248 & 0.0526 & 1.2412 & 1.4140 \\
\hline \multicolumn{6}{|c|}{$\underline{\text { B. Mutual utility }}\left(m_{i j}\right)$} \\
\hline CONSTANT & 6.1668 & 6.1659 & 0.0408 & 6.1010 & 6.2346 \\
\hline SAME GENDER & 1.0716 & 1.0716 & 0.0153 & 1.0462 & 1.0967 \\
\hline SAME GRADE & -3.0514 & -3.0510 & 0.0220 & -3.0882 & -3.0160 \\
\hline WHITE-WHITE & -0.6017 & -0.6016 & 0.0186 & -0.6322 & -0.5711 \\
\hline BLACK-BLACK & 1.1177 & 1.1175 & 0.0261 & 1.0750 & 1.1613 \\
\hline HISP-HISP & -1.4659 & -1.4655 & 0.0229 & -1.5033 & -1.4287 \\
\hline \multicolumn{6}{|c|}{ C. INDIRECT UTILITY AND POPULARITY $\left(v_{i j}\right)$} \\
\hline CONSTANT & -0.4705 & -0.4705 & 0.0071 & -0.4823 & -0.4587 \\
\hline SAME GENDER & -0.4074 & -0.4072 & 0.0069 & -0.4188 & -0.3962 \\
\hline SAME GRADE & 0.1136 & 0.1136 & 0.0095 & 0.0981 & 0.1293 \\
\hline WHITE-WHITE & 0.1856 & 0.1857 & 0.0090 & 0.1708 & 0.2004 \\
\hline BLACK-BLACK & 0.1372 & 0.1371 & 0.0081 & 0.1239 & 0.1507 \\
\hline HISP-HISP & -0.5067 & -0.5066 & 0.0111 & -0.5249 & -0.4886 \\
\hline
\end{tabular}

Estimated posterior distribution for the full structural model. The estimates are obtained with a 
Table D4-Model (3) in TABle 3

\begin{tabular}{|c|c|c|c|c|c|}
\hline & mean & median & std. dev. & 5 pctile & 95 pctile \\
\hline CONSTANT & -6.6500 & -6.6499 & 0.0189 & -6.6812 & -6.6191 \\
\hline MALE & -0.1517 & -0.1516 & 0.0105 & -0.1690 & -0.1349 \\
\hline WHITE & -0.1710 & -0.1709 & 0.0139 & -0.1938 & -0.1484 \\
\hline BLACK & 1.0451 & 1.0451 & 0.0048 & 1.0371 & 1.0530 \\
\hline HISP & 2.0990 & 2.0991 & 0.0235 & 2.0604 & 2.1375 \\
\hline INCOME & -2.0543 & -2.0542 & 0.0319 & -2.1075 & -2.0025 \\
\hline SAME GENDER & 0.2067 & 0.2068 & 0.0131 & 0.1848 & 0.2281 \\
\hline SAME GRADE & 2.3817 & 2.3814 & 0.0211 & 2.3469 & 2.4166 \\
\hline WHITE-WHITE & 1.0138 & 1.0136 & 0.0133 & 0.9921 & 1.0358 \\
\hline BLACK-BLACK & 1.6491 & 1.6489 & 0.0159 & 1.6233 & 1.6754 \\
\hline HISP-HISP & 0.3186 & 0.3184 & 0.0166 & 0.2914 & 0.3463 \\
\hline ATTRACTIVE i (Physical) & -2.3568 & -2.3567 & 0.0296 & -2.4057 & -2.3084 \\
\hline ATTRACTIVE j (Physical) & 2.5166 & 2.5163 & 0.0255 & 2.4750 & 2.5590 \\
\hline ATTRACTIVE i (Personality) & -0.4964 & -0.4964 & 0.0087 & -0.5108 & -0.4821 \\
\hline ATTRACTIVE j (Personality) & -1.0932 & -1.0930 & 0.0165 & -1.1205 & -1.0664 \\
\hline Income i - Income j (logs) & 0.8883 & 0.8883 & 0.0141 & 0.8654 & 0.9116 \\
\hline Income i + Income j (logs) & 1.0947 & 1.0947 & 0.0177 & 1.0660 & 1.1242 \\
\hline FRACTION WHITES & -1.7088 & -1.7087 & 0.0074 & -1.7210 & -1.6966 \\
\hline FRACTION BLACKS & 1.3416 & 1.3419 & 0.0128 & 1.3205 & 1.3625 \\
\hline FRACTION HISP & 0.8397 & 0.8397 & 0.0084 & 0.8260 & 0.8535 \\
\hline WHITE-WHITE * FRACTION WHITES & 4.3915 & 4.3908 & 0.0526 & 4.3059 & 4.4785 \\
\hline BLACK-BLACK * FRACTION BLACKS & 0.2528 & 0.2529 & 0.0061 & 0.2428 & 0.2627 \\
\hline HISP-HISP * FRACTION HISP & -1.6908 & -1.6907 & 0.0088 & -1.7053 & -1.6766 \\
\hline SCHOOL 1 & -0.2439 & -0.2439 & 0.0222 & -0.2807 & -0.2075 \\
\hline SCHOOL 2 & 1.7809 & 1.7807 & 0.0217 & 1.7450 & 1.8169 \\
\hline SCHOOL 3 & 1.7858 & 1.7858 & 0.0146 & 1.7616 & 1.8097 \\
\hline SCHOOL 4 & 1.9064 & 1.9061 & 0.0176 & 1.8780 & 1.9355 \\
\hline SCHOOL 5 & 2.2429 & 2.2428 & 0.0127 & 2.2221 & 2.2642 \\
\hline SCHOOL 6 & -1.4227 & -1.4226 & 0.0179 & -1.4523 & -1.3933 \\
\hline SCHOOL 7 & -0.2224 & -0.2223 & 0.0124 & -0.2428 & -0.2024 \\
\hline SCHOOL 8 & 0.2460 & 0.2457 & 0.0232 & 0.2084 & 0.2842 \\
\hline SCHOOL 9 & -2.7969 & -2.7967 & 0.0425 & -2.8673 & -2.7275 \\
\hline SCHOOL 10 & 0.8911 & 0.8914 & 0.0262 & 0.8480 & 0.9341 \\
\hline SCHOOL 11 & -1.0609 & -1.0608 & 0.0206 & -1.0949 & -1.0270 \\
\hline SCHOOL 12 & 0.9857 & 0.9859 & 0.0206 & 0.9516 & 1.0191 \\
\hline SCHOOL 13 & 2.9091 & 2.9085 & 0.0523 & 2.8237 & 2.9954 \\
\hline
\end{tabular}

Estimated posterior distribution for the full structural model. The estimates are obtained with a sample of 100000 parameter simulations, and 5000 network simulations for each parameter proposal. 
Table D5-Model (6) in TABle 3

\begin{tabular}{|c|c|c|c|c|c|}
\hline & mean & median & std. dev. & 5 pctile & 95 pctile \\
\hline \multicolumn{6}{|c|}{ A. DiRECT UTILITY $\left(u_{i j}\right)$} \\
\hline CONSTANT & -5.8070 & -5.8070 & 0.0068 & -5.8185 & -5.7958 \\
\hline MALE & 0.2350 & 0.2348 & 0.0081 & 0.2212 & 0.2483 \\
\hline WHITE & 0.3023 & 0.3023 & 0.0120 & 0.2826 & 0.3219 \\
\hline BLACK & 1.1801 & 1.1801 & 0.0028 & 1.1755 & 1.1847 \\
\hline HISP & 2.0295 & 2.0297 & 0.0197 & 1.9967 & 2.0616 \\
\hline INCOME & -1.4645 & -1.4645 & 0.0259 & -1.5072 & -1.4216 \\
\hline SAME GENDER & 0.7644 & 0.7644 & 0.0093 & 0.7491 & 0.7797 \\
\hline SAME GRADE & 2.1800 & 2.1798 & 0.0162 & 2.1533 & 2.2067 \\
\hline WHITE-WHITE & 0.2739 & 0.2739 & 0.0063 & 0.2637 & 0.2839 \\
\hline BLACK-BLACK & 0.9405 & 0.9405 & 0.0098 & 0.9246 & 0.9570 \\
\hline HISP-HISP & -0.1394 & -0.1395 & 0.0098 & -0.1554 & -0.1226 \\
\hline BEAUTY i & -1.9430 & -1.9432 & 0.0258 & -1.9855 & -1.9001 \\
\hline BEAUTY j & 1.2609 & 1.2609 & 0.0121 & 1.2412 & 1.2812 \\
\hline PERSONALITY i & -0.1631 & -0.1633 & 0.0057 & -0.1725 & -0.1533 \\
\hline PERSONALITY j & -0.3939 & -0.3939 & 0.0135 & -0.4164 & -0.3716 \\
\hline Income i - Income j & 0.7403 & 0.7401 & 0.0134 & 0.7178 & 0.7621 \\
\hline Income i + Income j & 0.6892 & 0.6894 & 0.0149 & 0.6643 & 0.7136 \\
\hline FRACTION WHITES & -1.6126 & -1.6124 & 0.0060 & -1.6228 & -1.6029 \\
\hline FRACTION BLACKS & 1.9618 & 1.9618 & 0.0063 & 1.9514 & 1.9722 \\
\hline FRACTION HISP & 0.7731 & 0.7731 & 0.0066 & 0.7623 & 0.7839 \\
\hline WHITE-WHITE * FRACTION WHITE & 2.3272 & 2.3271 & 0.0340 & 2.2719 & 2.3837 \\
\hline BLACK-BLACK $*$ FRACTION BLACKS & 0.2516 & 0.2515 & 0.0066 & 0.2410 & 0.2624 \\
\hline HISP-HISP $*$ FRACTION HISP & -1.1400 & -1.1399 & 0.0059 & -1.1494 & -1.1302 \\
\hline SCHOOL 1 & -0.1335 & -0.1336 & 0.0163 & -0.1603 & -0.1067 \\
\hline SCHOOL 2 & 1.4996 & 1.4994 & 0.0169 & 1.4722 & 1.5276 \\
\hline SCHOOL 3 & 1.8785 & 1.8785 & 0.0102 & 1.8620 & 1.8954 \\
\hline SCHOOL 4 & 1.3724 & 1.3724 & 0.0106 & 1.3553 & 1.3898 \\
\hline SCHOOL 5 & 1.6828 & 1.6827 & 0.0088 & 1.6686 & 1.6973 \\
\hline SCHOOL 6 & -1.0683 & -1.0679 & 0.0128 & -1.0902 & -1.0481 \\
\hline SCHOOL 7 & -0.9817 & -0.9815 & 0.0280 & -1.0285 & -0.9346 \\
\hline SCHOOL 8 & -0.5932 & -0.5929 & 0.0203 & -0.6274 & -0.5602 \\
\hline SCHOOL 9 & 0.2444 & 0.2442 & 0.0109 & 0.2267 & 0.2624 \\
\hline SCHOOL 10 & -1.1949 & -1.1948 & 0.0168 & -1.2230 & -1.1679 \\
\hline SCHOOL 11 & -2.3824 & -2.3821 & 0.0379 & -2.4446 & -2.3196 \\
\hline SCHOOL 12 & 1.2316 & 1.2318 & 0.0248 & 1.1911 & 1.2720 \\
\hline SCHOOL 13 & -1.4722 & -1.4719 & 0.0203 & -1.5061 & -1.4389 \\
\hline SCHOOL 14 & 1.8479 & 1.8480 & 0.0084 & 1.8339 & 1.8617 \\
\hline SCHOOL 15 & 0.5666 & 0.5663 & 0.0301 & 0.5176 & 0.6166 \\
\hline \multicolumn{6}{|c|}{$\underline{\text { B. Mutual utility }}\left(m_{i j}\right)$} \\
\hline CONSTANTm & 5.3139 & 5.3137 & 0.0257 & 5.2721 & 5.3572 \\
\hline SAME GENDERm & 1.1539 & 1.1536 & 0.0088 & 1.1397 & 1.1688 \\
\hline SAME GRADEm & -3.0575 & -3.0575 & 0.0158 & -3.0831 & -3.0317 \\
\hline WHITE-WHITEm & -0.4960 & -0.4959 & 0.0120 & -0.5162 & -0.4766 \\
\hline BLACK-BLACKm & 0.7067 & 0.7068 & 0.0178 & 0.6771 & 0.7362 \\
\hline HISP-HISPm & -1.4639 & -1.4639 & 0.0120 & -1.4839 & -1.4442 \\
\hline \multicolumn{6}{|c|}{ C. INDIRECT UTILITY AND POPULARITY $\left(v_{i j}\right)$} \\
\hline CONSTANTv & -0.4308 & -0.4309 & 0.0048 & -0.4386 & -0.4230 \\
\hline SAME GENDERv & -0.3987 & -0.3987 & 0.0045 & -0.4061 & -0.3914 \\
\hline SAME GRADEv & 0.3266 & 0.3266 & 0.0072 & 0.3148 & 0.3384 \\
\hline WHITE-WHITEv & 0.2978 & 0.2978 & 0.0042 & 0.2909 & 0.3047 \\
\hline BLACK-BLACKv & 0.1202 & 0.1203 & 0.0088 & 0.1057 & 0.1343 \\
\hline HISP-HISPv & -0.2859 & -0.2860 & 0.0059 & -0.2958 & -0.2759 \\
\hline
\end{tabular}

Estimated posterior distribution for the full structural model. The estimates are obtained with a sample of 20000 parameter simulations, and 10000 network simulations for each parameter proposal. 
Table D6-Model (5) in Table 3

\begin{tabular}{|c|c|c|c|c|c|}
\hline & mean & median & std. dev. & 5 pctile & 95 pctile \\
\hline \multicolumn{6}{|c|}{ A. DiRECT UTILITY $\left(u_{i j}\right)$} \\
\hline CONSTANT & -7.2182 & -7.2151 & 0.0329 & -7.2761 & -7.1685 \\
\hline MALE & -0.2718 & -0.2717 & 0.0301 & -0.3208 & -0.2232 \\
\hline WHITE & 0.0440 & 0.0445 & 0.0455 & -0.0286 & 0.1136 \\
\hline BLACK & 0.7074 & 0.7049 & 0.0138 & 0.6880 & 0.7323 \\
\hline HISP & 1.4590 & 1.4588 & 0.0213 & 1.4250 & 1.4946 \\
\hline INCOME & -1.8738 & -1.8740 & 0.0279 & -1.9215 & -1.8279 \\
\hline SAME GENDER & 0.3154 & 0.3153 & 0.0156 & 0.2898 & 0.3406 \\
\hline SAME GRADE & 2.5185 & 2.5173 & 0.0297 & 2.4713 & 2.5689 \\
\hline WHITE-WHITE & 0.9959 & 0.9832 & 0.0534 & 0.9271 & 1.0975 \\
\hline BLACK-BLACK & 1.5347 & 1.5251 & 0.0437 & 1.4755 & 1.6159 \\
\hline HISP-HISP & 0.7130 & 0.7030 & 0.0530 & 0.6427 & 0.8099 \\
\hline BEAUTY i & -1.9291 & -1.9295 & 0.0266 & -1.9732 & -1.8841 \\
\hline BEAUTY j & 2.7615 & 2.7616 & 0.0242 & 2.7218 & 2.8005 \\
\hline PERSONALITY i & -0.8646 & -0.8571 & 0.0401 & -0.9359 & -0.8087 \\
\hline PERSONALITY j & -0.6361 & -0.6332 & 0.0238 & -0.6817 & -0.6017 \\
\hline Income i - Income j & 0.9938 & 0.9943 & 0.0141 & 0.9695 & 1.0169 \\
\hline Income $\mathrm{i}+$ Income $\mathrm{j}$ & 0.8977 & 0.8979 & 0.0164 & 0.8704 & 0.9243 \\
\hline FRACTION WHITES & -1.5748 & -1.5614 & 0.0661 & -1.6958 & -1.4910 \\
\hline FRACTION BLACKS & 0.7645 & 0.7742 & 0.0534 & 0.6684 & 0.8375 \\
\hline FRACTION HISP & 1.0078 & 1.0023 & 0.0319 & 0.9660 & 1.0638 \\
\hline WHITE-WHITE * FRACTION WHITE & 4.7269 & 4.7281 & 0.0509 & 4.6417 & 4.8081 \\
\hline BLACK-BLACK * FRACTION BLACKS & 0.1172 & 0.1171 & 0.0125 & 0.0974 & 0.1382 \\
\hline HISP-HISP $*$ FRACTION HISP & -1.3872 & -1.3915 & 0.0297 & -1.4288 & -1.3364 \\
\hline SCHOOL 1 & -0.4403 & -0.4408 & 0.0232 & -0.4783 & -0.4007 \\
\hline SCHOOL 2 & 2.4641 & 2.4648 & 0.0204 & 2.4303 & 2.4969 \\
\hline SCHOOL 3 & 1.3139 & 1.3041 & 0.0418 & 1.2578 & 1.3919 \\
\hline SCHOOL 4 & 2.4282 & 2.4233 & 0.0356 & 2.3778 & 2.4915 \\
\hline SCHOOL 5 & 2.8177 & 2.8181 & 0.0191 & 2.7867 & 2.8487 \\
\hline SCHOOL 6 & -1.7375 & -1.7362 & 0.0249 & -1.7802 & -1.6982 \\
\hline SCHOOL 7 & -0.7972 & -0.7982 & 0.0387 & -0.8597 & -0.7306 \\
\hline SCHOOL 8 & -1.6076 & -1.6071 & 0.0584 & -1.7031 & -1.5125 \\
\hline SCHOOL 9 & 0.4031 & 0.4053 & 0.0193 & 0.3675 & 0.4317 \\
\hline SCHOOL 10 & -0.9558 & -0.9558 & 0.0405 & -1.0210 & -0.8891 \\
\hline SCHOOL 11 & -2.5207 & -2.5209 & 0.0388 & -2.5865 & -2.4563 \\
\hline SCHOOL 12 & 0.7806 & 0.7750 & 0.0429 & 0.7171 & 0.8594 \\
\hline SCHOOL 13 & -1.4684 & -1.4657 & 0.0440 & -1.5433 & -1.3997 \\
\hline SCHOOL 14 & 0.0815 & 0.0818 & 0.0257 & 0.0388 & 0.1237 \\
\hline SCHOOL 15 & 3.6894 & 3.6896 & 0.0506 & 3.6055 & 3.7699 \\
\hline
\end{tabular}

Estimated posterior distribution for the full structural model. The estimates are obtained with a sample of 20000 parameter simulations, and 10000 network simulations for each parameter proposal. 